Biologie Aujourd'hui, ??? (?), ???-??? (2016)

(C) Société de Biologie, 2016

DOI: $10.1051 /$ jbio/2016???

\title{
Fourmis : une chimiothèque de nouveaux anticancéreux
}

\author{
Angélique Vétillard et Wafa Bouzid \\ Équipe Venins et Activités Biologiques (VAcBio), EA 4357, PRES-Université de Toulouse, Institut Universitaire \\ Jean-François Champollion, place de Verdun, 81000 Albi, France
}

Auteur correspondant: Angélique Vétillard, angelique.vetillard@univ-jfc.fr

accepté le 7 juin 2016

Résumé - La composition des venins est très compliquée à déterminer à cause de leur complexité et de leur diversité moléculaire, cependant l'étude transcriptomique des glandes à venin de la fourmi Tetramorium bicarinatum révèle une grande diversité fonctionnelle des peptides/protéines qui pourraient être produits par ces glandes. Ces molécules peptidiques correspondent pour partie à des toxines connues mais de nombreuses séquences ne sont répertoriées dans aucune base de données et pourraient correspondre à des peptides de venins originaux non décrits auparavant. Nos travaux étaient les premiers à rapporter le séquençage du transcriptome de la glande à venin chez une fourmi, d'abord par la technique de la SSH (Suppression Subtractive Hybridization), puis par une technique de séquençage profond permettant de progresser dans le degré d'informations que renferme le transcriptome (Illumina). Nos expériences montrent clairement que l'extrait de venin brut induit l'apoptose de certaines lignées de cellules tumorales résistantes à tous les traitements actuels et l'analyse en spectrométrie de masse nous a orientés vers une molécule dont la structure nous est encore inconnue. L'identification et la caractérisation du mécanisme d'action de la molécule responsable de cette cytotoxicité constituent donc un espoir de découvrir de nouvelles stratégies thérapeutiques dans la lutte contre cette pathologie encore aujourd'hui incurable. D'une manière générale, l'extraordinaire diversité taxonomique des fourmis laisse présager d'une grande diversité de leurs toxines (Hoffman, 2010). La probabilité de découvrir de nouvelles molécules d'intérêt pour l'homme n'en est que renforcée.

Mots clés : Fourmi Tetramorium bicarinatum / toxines de venin / allergènes d'hyménoptères / Séquençage EST / séquençage de novo Illumina

Abstract - Ants: a chemical library of anticancer molecules.

Animal venoms are complex mixtures containing simple organic molecules, proteins, peptides, and other bioactive elements with extraordinary biological properties associated with their ability to act on a number of molecular receptors in the process of incapacitating their target organisms. In such a context, arthropod venoms are invaluable sources of bioactive substances with therapeutic interest but the limited availability of some venom such as those from ants, has restricted the potential that these biomolecules could represent. We investigated for the first time transcriptomic expression from the ant species Tetramorium bicarinatum. Four hundred randomly selected clones from cDNA libraries were sequenced and a total of 374 expressed sequence tags (ESTs) were generated. Based on the results of BLAST searches, these sequences were clustered and assembled into 269 contigs. About $72 \%$ (269) of these matched BLASTx hits with an interesting diversity and unusual abundance of cellular transcripts $(48 \%)$ related to gene and protein expression reflecting the specialization of this tissue. In addition, transcripts encoding transposases were relatively highly expressed $(14 \%)$. It may be that transposable elements are present and that their presence accounts for some of the variation in venom toxins. About twenty per cent of the ESTs were categorized as putative toxins, the major part represented by allergens (48\% of the total venom toxins) such as pilosulin 5, sol i 3 and Myp p I and II. 
Several contigs encoding enzymes, including zinc-metalloproteases (17\%) that are likely involved in the processing and activation of venom proteins/peptides, were also identified from the library. In addition, a number of sequences $(8 \%)$ had no significant similarity to any known sequence which indicates a potential source of for the discovery of new toxins. In order to provide a global insight on the transcripts expressed in the venom gland of the Brazilian ant species Tetramorium bicarinatum and to unveil the potential of their products, high-throughput expressed sequence tags were generated using Illumina paired-end sequencing technology. A total of 212371758 pairs of quality-filtered, 100-base-pair Illumina reads were obtained. The de novo assemblies yielded 36042 contigs for which 27873 have at least one predicted ORF among which $59.77 \%$ produce significant hits in the available databases. The investigation of the read mapping toxin class revealed and confirmed a high diversification with the major part consistent with the classical hymenopteran venom protein signature represented by venom allergen $(\mathbf{3 3 . 3 \%})$ followed by a diverse toxin-expression profile including several distinct isoforms of phospholipase $\mathbf{A}_{1}$ and $\mathbf{A}_{2}$, venom serine protease, hyaluronidase, protease inhibitor and secapin. Moreover, our results revealed for the first time the presence of toxin-like peptides that have been previously identified from unrelated venomous animals such as waprin-like (snakes) and agatoxins (spiders and conus). These studies provide a first insight of the gene expression scenario of the venom gland of $T$. bicarinatum which might contribute to acquiring a more comprehensive view about the origin and functional diversity of venom proteins of this ant. Based on such results, we conducted cytotoxic tests from the crude venom of $T$. bicarinatum ant and reported toxic effect on tumoral cells lines from one of the fifth of the most frequently occurring cancers with a 3 -year survival rate of only $30 \%$. In such a context, new therapeutic strategies are essential and the discovery of new molecules in ant venom could be one possible avenue. Thus our project aims to characterize, from the crude venom of $T$. bicarinatum, the molecule(s) which have potential anti-cancerous toxicity as well as their mechanisms of action.

Key words: Tetramorium bicarinatum ant / venom toxins / hymenopteran allergens / ESTs library / Illumina technology

\section{Les venins de fourmis, une richesse chimique encore inexplorée}

3 Les venins animaux, par leur richesse biochimique et la diversité de leurs cibles moléculaires, sont une source très importante de nouvelles molécules dont les applications potentielles sont nombreuses, en particulier dans le domaine médical (Lewis \& Garcia, 2003; King, 2011). Même si la recherche sur le développement d'anti-venins reste une préoccupation majeure, depuis plusieurs années la communauté scientifique s'intéresse, en effet, à identifier, dans les venins, de nouvelles molécules bioactives susceptibles d'intéresser le domaine médical mais aussi pharmacologique et agronomique. La recherche de molécules bioactives d'intérêt sociétal s'est d'abord orientée vers les venins de serpents, en raison de leur action foudroyante et des dommages corporels que peut engendrer une morsure, mais également en raison de l'abondance du venin chez ces animaux. Depuis une trentaine d'années, de nombreux travaux sont développés sur les venins d'insectes, ce que l'on peut en partie expliquer par les avancées technologiques rendant accessible l'étude de très petites quantités de venins et la caractérisation de molécules à l'état de traces. Si parmi les études portant sur les venins d'hyménoptères, les abeilles et les guêpes sont des modèles récurrents dans la littérature, les études sur les venins de fourmis sont encore rares.

Aujourd'hui, seul le venin de quelques espèces a été exploré, avec à chaque fois la mise en évidence de toxines originales (Davies et al., 2004). Pourtant, les fourmis (Formicidae) sont ubiquistes et quantitativement dominantes au sein des milieux colonisés (Floren et al., 2002; Moreau et al., 2006). La myrmécofaune renferme ainsi la plus importante richesse spécifique parmi les insectes sociaux avec plus de 15000 espèces recensées dans la base de données AntWeb. L'une des premières expressions de cette richesse est l'existence de différences importantes dans leur écologie, et notamment selon leur mode de vie. Les fourmis sont ainsi présentes sur tous les territoires et au regard de la grande diversité dans leur écologie, leurs sécrétions chimiques, adaptées notamment pour attaquer, se défendre et communiquer chimiquement, représentent une richesse de diversification offrant des perspectives de nouvelles découvertes très importantes dans ce groupe zoologique. Les investigations déjà menées sur le venin des fourmis ont d'ailleurs révélé un mélange de molécules d'une grande complexité et 


$$
\text { “jbio160021" - 2016/8/16 - 15:51 - page } 3 \text { - \#3 }
$$
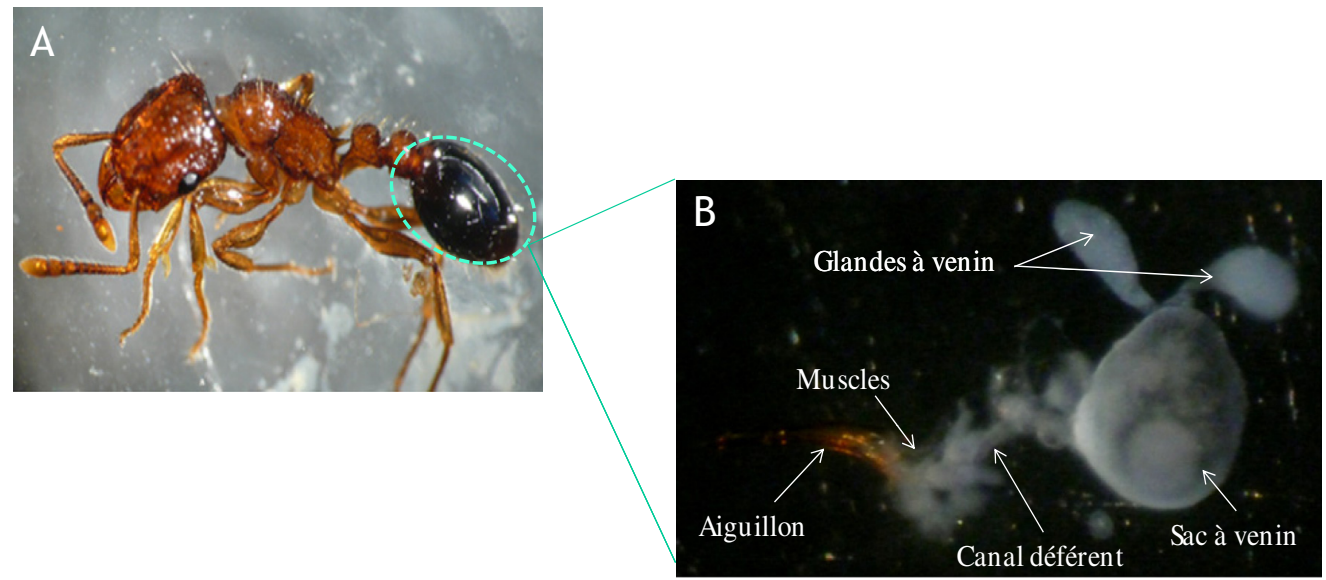

Fig. 1. Appareil vulnérant de la fourmi Tetramorium bicarinatum.

\begin{abstract}
diversité chimique représentant une source précieuse de composés biologiquement actifs. La compréhension du mode d'action des toxines des venins et la synthèse d'analogues impliquent notamment une connaissance précise de leur structure chimique et de leur conformation tridimensionnelle. Dans ce sens, la stratégie de recherche de l'équipe VAcBio combine des approches intégrées complémentaires dans l'identification de nouvelles molécules d'un grand intérêt pour le développement de nouveaux médicaments capables d'agir directement et spécifiquement sur la physiologie cellulaire.
\end{abstract}

\section{Elaborer une banque transcriptomique de la glande à venin de Tetramorium bicarinatum : une stratégie pour révéler de nouvelles molécules médicamenteuses}

L'espèce de fourmi exotique terricole sur laquelle nous travaillons, Tetramorium bicarinatum, a été sélectionnée sur la base de caractéristiques comportementales étayées par l'expertise de Jérôme Orivel, chercheur spécialiste des fourmis. T. bicarinatum, Myrmicinae de la tribu des Tetramorinii appartenant à la famille des Formicidae, est une fourmi de petite taille (3,4 à 4,5 mm) (Figure 1A), d'abord décrite sous le nom de Myrmica bicarinata par Nylander en 1846 Son appareil vulnérant est composé notamment de deux glandes à venin qui débouchent dans le réservoir à venin (Figure $1 \mathrm{~B})$. Ce réservoir ou sac à venin est relié à l'aiguillon par le canal déférent dans lequel est déversé le venin supposé renfermer un cocktail varié de molécules, une composition de nature majoritairement peptidique et très compliquée à déterminer, en réponse à la diversité biologique de son habitat exotique.
D'une manière générale, la plupart des études sur les venins correspondent à une caractérisation directe biochimique/protéique plutôt qu'à des études de génomique ou de transcriptomique. Pourtant, les quelques études de transcriptomique se sont avérées très utiles pour révéler la complexité des composants des venins et des processus cellulaires sous-jacents (Liang, 2008; Ruiming et al., 2010). Ces approches ont aussi montré leur efficacité dans la découverte de nouvelles toxines jusqu'alors non révélées par des méthodes directes. Les résultats générés sont révélateurs des processus biologiques mis en œuvre dans les cellules de la glande à venin, mais apportent également une vue générale, jamais investiguée par ailleurs, sur la dynamique moléculaire au niveau de la glande à venin.

Nos travaux consistaient à répertorier des gènes qui s'exprimaient différentiellement dans les glandes à venin et pour la première fois chez une espèce de fourmi exotique, Tetramorium bicarinatum, fourmi sélectionnée eu égard à sa diversité biologique et à la puissance de son venin contre l'homme et les vertébrés en général. L'intérêt d'identifier les molécules qui composent ce venin réside notamment dans leurs éventuelles vertus thérapeutiques. Dans le domaine de lutte contre le cancer, un article a d'ailleurs montré que le venin d'une espèce de fourmis du genre Samsum induit l'apoptose d'une lignée cellulaire de cancer mammaire (Badr et al., 2012).

Les collections de marqueurs de séquences exprimées représentent ainsi un point de départ indéniable pour l'identification de gènes et donc de nouvelles molécules médicamenteuses, notamment chez les organismes n'ayant pas de génome de référence.

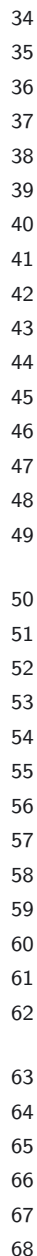

34 


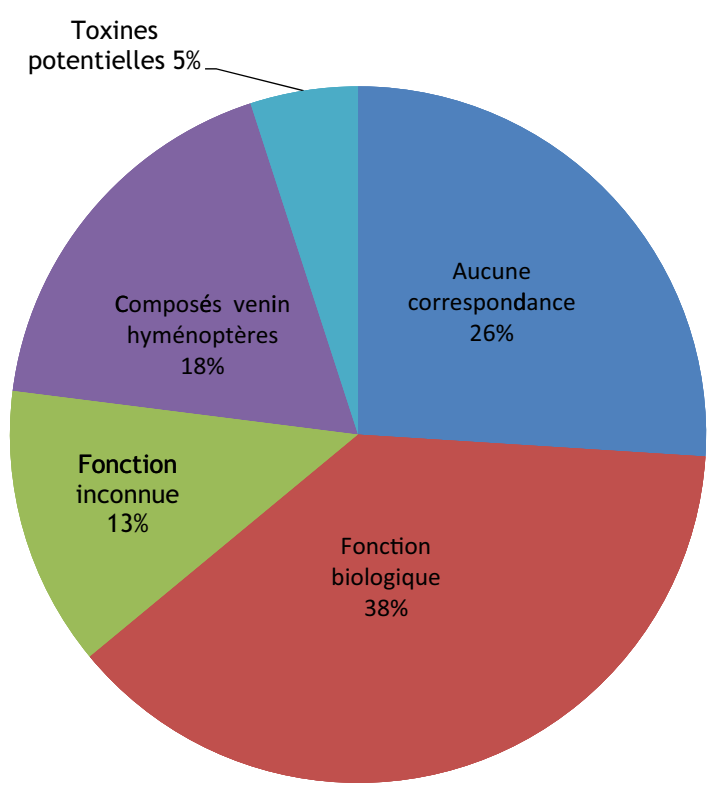

Fig. 2. Répartition des transcrits identifiés dans la glande à venin de Tetramorium bicarinatum.

\section{Le premier transcriptome de la glande à venin chez une fourmi a été séquencé dans l'équipe VAcBio (Bouzid et al., 2013) par la technique SSH « Suppressive Subtractive Hybridization »}

Les analyses des séquences ont révélé une grande diversité fonctionnelle des peptides/protéines dérivés qui pourraient être produits par ces glandes, avec $38 \%$ impliqués dans des processus cellulaires, $18 \%$ correspondant à des composés caractéristiques des venins d'hyménoptères et $5 \%$ à des candidats potentiels de toxines des venins (Figure 2).

L'analyse des séquences caractéristiques de transcrits relatifs aux processus cellulaires des glandes à venin rapporte une importante représentation d'un groupe de méthyltransférases, enzymes catalytiques dotées d'un rôle fondamental dans la régulation de la division cellulaire et la méthylation des molécules d'ADN. Un groupe de transposases est également fortement représenté (16\%), suggérant ainsi la présence probable d'éléments transposables souvent à l'origine de mutations génétiques et par conséquent de la diversification des toxines (Figure 3).

Parmi les séquences caractéristiques de composés de venins d'hyménoptères et de possibles toxines, émerge un grand nombre de molécules impliquées dans le système immunitaire, les processus inflammatoires et la division cellulaire. Au sein des séquences

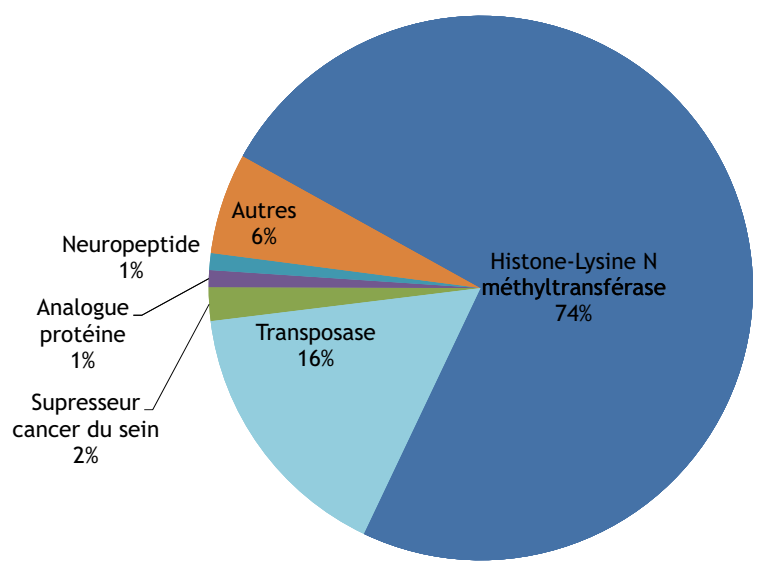

Fig. 3. Répartition de l'abondance des transcrits relatifs aux processus cellulaires de la glande à venin de Tetramorium bicarinatum.

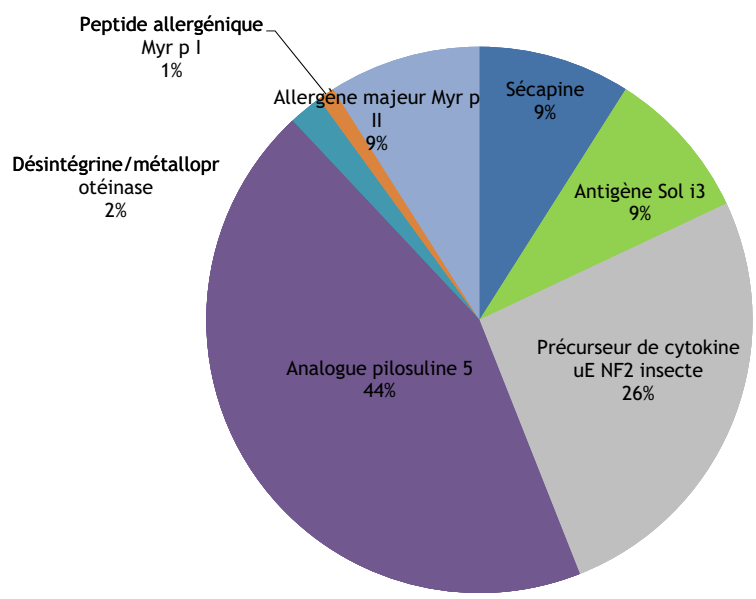

Fig. 4. Répartition de l'abondance des transcrits relatifs à de possibles toxines exprimées dans la glande à venin de Tetramorium bicarinatum.

assimilées à de possibles toxines, le groupe majoritaire $(44 \%)$ correspond à des polypeptides allergènes de type «pilosuline », suivi d'un groupe de précurseurs de cytokines relativement abondant au sein de la banque (26\%). On trouve également des métalloprotéinases ( $2 \%$ ), enzymes identifiées dans le venin de serpents et largement décrites pour leur effet anti-tumoral (Zhu et al., 2010) (Figure 4).

Enfin, une proportion non négligeable de nos séquences $(26 \%)$ (Figure 2) n'a pas été identifiée, et ce malgré plusieurs bases de données sollicitées. Ces séquences, dotées pour la plupart de séquences signal et de pattern cystéine, correspondraient par hypothèse à des peptides de venins originaux n'ayant pas été 


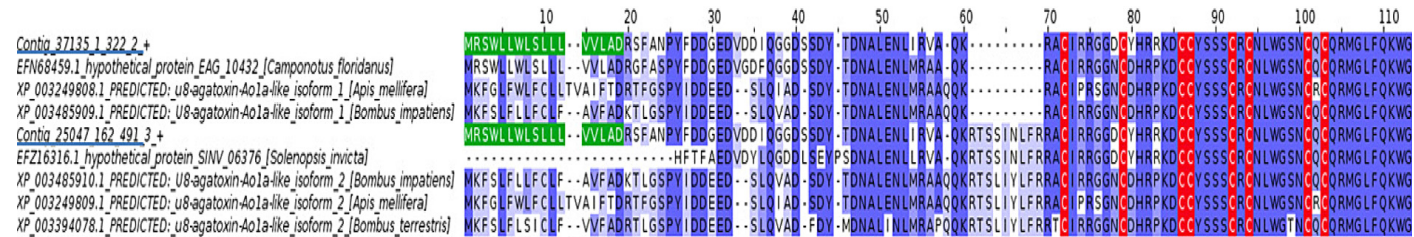

Fig. 5. Alignement de séquences d'acides aminés de l'agatoxine-like de Tetramorium bicarinatum (contig 37135 et 25047 ) avec des agatoxines d'autres Hyménoptères. Le degré de conservation des acides aminés est indiqué par un dégradé de bleu. Les cystéines conservées sont surlignées en rouge et la séquence signal en vert.

1 décrits auparavant et pouvant être spécifiques à notre espèce de fourmis. Ces résultats s'avèrent très prometteurs quant à la probabilité de découvrir de nouvelles molécules d'intérêt sociétal, puisque ces séquences collectionnées dans la «Gene Ontology Database » pourraient correspondre à des molécules jusqu'alors inconnues. La technique utilisée, à savoir la SSH (Suppressive Subtractive Hybridization) nous a permis de réaliser un premier inventaire des transcrits présents dans la glande à venin de $T$. bicarinatum. Les séquences de transcrits générés nous ont servi de «banque locale » de séquences, sur la base de laquelle nous avons conduit un séquençage transcriptomique (RNA-seq) de novo à haut débit (Illumina) permettant une description exhaustive et quantitative.

\section{Séquençage nouvelle génération (Illumina) du transcriptome de la glande à venin (Bouzid et al., 2014)}

Nous avons effectué un séquençage aléatoire entier du transcriptome de la glande à venin via la technique RNA seq de novo à très haut débit. Cette méthode permet non seulement l'acquisition simultanément de plus de 3 milliards de séquences de 100 paires de bases de longueur, mais aussi l'assemblage des séquences générées même en absence de génome de référence, ce qui constitue un atout majeur pour les organismes non modèles tels que $T$. bicarinatum.

De ce séquençage haut débit, des informations complémentaires à celles déjà obtenues révèlent la présence de séquences correspondant à de nombreuses toxines putatives habituellement décrites chez les serpents et les araignées telle que l'agatoxine (Figure 5) appartenant aux peptides neurotoxiques avec potentiel thérapeutique. Ils sont notamment très utilisés, dans le traitement des maladies des canaux ioniques, pour l'étude de la structure et de l'activité de ces derniers (Pringos et al., 2011). Ces données ont également montré l'existence de peptides tels que la phospholipase A2 (Figure 6), de plus en plus documentés pour leur effet anti-tumoral et leur utilisation potentielle comme transporteurs d'antigènes tumoraux pour

la conception de vaccins contre les cancers (Almunia et al., 2013).

Ces données serviront de base et seront croisées avec des données générées par des études de protéomique et confirmeront ainsi les séquences prédites par les techniques de séquençage transcriptomiques. Malgré la difficulté de réaliser cette technique, tributaire de la quantité du venin et sa qualité d'extraction, une première analyse a été réalisée et a permis de dégager un nombre de peptides «nouveaux » non révélés jusque-là.

Le premier séquençage du transcriptome de la glande à venin d'une fourmi a été réalisé dans notre équipe, chez l'espèce exotique Tetramorium bicarinatum et a révélé de nouvelles molécules potentiellement à l'origine de la bioactivité anticancéreuse de son venin.

L'intérêt d'identifier les molécules du venin réside notamment dans leurs éventuelles vertus thérapeutiques. Dans ce sens, ce séquençage a, en effet, été très informatif puisque l'identification des séquences transcrites dans les cellules des glandes à venin a été utilisée notamment comme base décisionnelle pour le ciblage des tests de bioactivité, et nous a orientés sur la piste d'une lignée cancéreuse qui s'est avérée très sensible au venin.

\section{Bioactivité anticancéreuse du venin de Tetramorium bicarinatum et identification de nouvelles cibles thérapeutiques dans la lutte contre le cancer}

Si l'application clinique de certaines toxines a 72 été possible pour certaines pathologies telles que 73 l'hypertension, la douleur chronique, le diabète et 74 la thrombose (King, 2011), aucune molécule obte- 75 nue à partir de venins d'animaux n'est utilisée au- 76 jourd'hui en thérapie anticancéreuse. Néanmoins, cer- 77 taines études rapportent des effets antiprolifératifs de 78 certains venins in vitro mais également in vivo chez la 79 souris. Le venin de scorpion est capable d'inhiber la 80 prolifération in vitro de cellules de gliome ainsi que la 81 


T.bicarinatum_PLA2-like/1-216
solP80003V1-142
splP00630/1-167
sp|P82971V1-136
sp|Q7M416V1-136
sp|POCQL9V1-239
sp|P59888V1-167
sp|PODKU2V1-145
sp|Q6PXPOV1-157

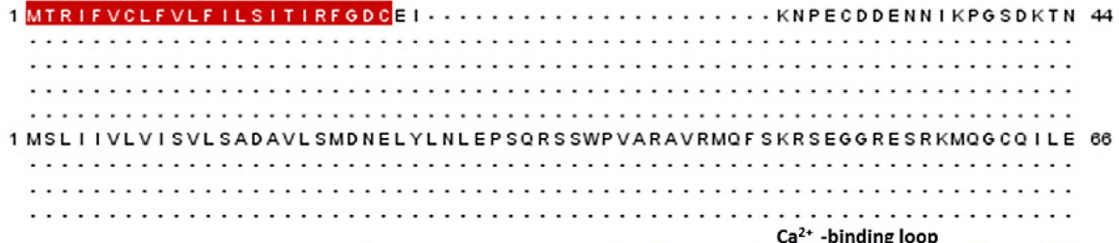

T.bicarinatum_PLA2-like/1-216 SolP80003V1-142 splpo0630/1-167 sop|P829711/1-136 solQ7M4/6V1-136 sp|POCQL $9 V 1-239$ sp|P59888V1-167 so|PODKU2V1-145 SPIQ6PXPOV $1-15$

T.bicarinatum_PLA2-like/1-216 sop|P80003V1-142 splpo0630/1-167 sop s.plP82971V 1-136 sp|Q7M4/6V1-136 SO|POCRL $9 / 1-239$ sp|P59888V1-167 so|PODKU2V1-145 s:D|Q6PXPOV1-157

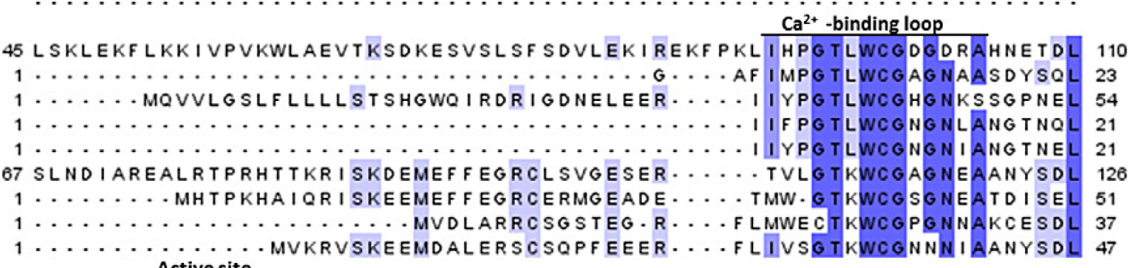
$1 \ldots \ldots \ldots \ldots$ MVKRVSKEEMDALERSCSQPFEEER . . F FLIVSOTIKWCONNN IAANYSDL 37

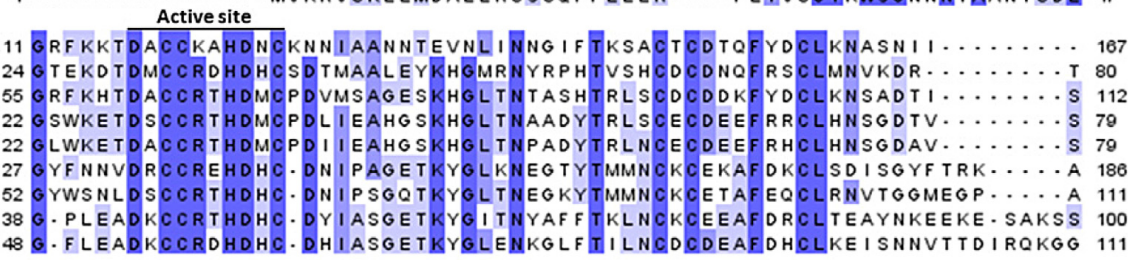
T.bicarinatum_PLA2-like/1-216 s.p|P80003V1-142 solP00630/1-167 solP82971V1-136 SolQ7M4I6V1-136 50107Mt/2 sp|POCRL $9 V 1-239$ sp|P59888V1-167 sp|PODKU2V1-145

168 ASEIGVTYFINLGPQCFKCKCPEKGCDLKDG . TECKDY......... CKKYEWINNRRF... 210 81 ADLVGMTYF TVLKISCFELE.EGEGC.VDNNF SQQCTKSE IMPVAKLVSAAPYQAQAETQSGEG 113 SYF VGKMYF NL I DTKCYKLEHPVTGCGERTE. . GRELHY .... TVDKSKPKVYQWF DLRKY... 167 80 AGF VGRTYFTVLHTQCFRLDYPIVKCKVKSTILHRSKCY.... DFETFAPKKYQWF DVLQY... 130 80 AAF VGRTYFT ILGTQ CFRLDYPIVKCKVKSTILRECKEY..... EF DTNAPQKYQWF DVLSY... 187 VSAVKF TYFTLYGNGCYNVKCENGRSPSNE..... CPNG . . . V VAEYTGETGLG. AKVINFGK. 230 112 AGF VRKTYFDLYGNG YNVQCPSORRLARSE... ECPDG....VATYTGEAGYGAWAINKLNG. 101 TKRLONFYFGTYSPECYVVTCNSKRSGRDAG .... 112 TKRLQNF

Fig. 6. Alignement de séquences d'acides aminés de la phospholipase A2-like de Tetramorium bicarinatum avec des phospholipases A2 d'autres Hyménoptères, de scorpion et de lézard indiqués par leur code d'accès dans GenBank. Le degré de conservation des acides aminés est indiqué par un dégradé de bleu. La séquence signal prédite est surlignée en rouge. Les régions soulignées indiquent le site de fixation de la boucle calcium-dépendante et le site actif de l'enzyme en accord avec la numérotation des acides aminés de la PLA2 de pancréas bovin (White et al., 1990).

croissance tumorale chez des souris xénogreffées avec une lignée de gliome malin (Wang \& Ji, 2005; Fu et al., 2007). Le venin d'abeille s'est avéré capable d'induire l'apoptose in vitro de cellules leucémiques (Moon et al., 2006) tout comme celui d'une anémone de mer déclenchant une apoptose spécifique de lignées de cancer mammaire (Ramezanpour et al., 2013). Dans certains cas, des toxines ayant une activité anti-tumorale ont pu être identifiées à partir de venins bruts, c'est le cas de la mélittine présente dans le venin d'abeille et connue pour avoir un effet anti-angiogénique (Shin et al., 2013).

En ce qui concerne les fourmis, une étude montre que le venin d'un type de fourmis est hautement toxique pour une lignée de cancer mammaire (Badr et al., 2012) alors que les travaux que nous menons avec le venin de Tetramorium bicarinatum font état d'un effet apoptotique sur une lignée de cellules tumorales jusqu'alors particulièrement résistantes à tous les traitements actuels.
L'identification et la caractérisation du mécanisme d'action de la molécule responsable de cette cytotoxicité constituent donc un espoir de découvrir de nouvelles stratégies thérapeutiques dans la lutte contre cette pathologie encore aujourd'hui incurable. D'une manière générale, l'extraordinaire diversité taxonomique des fourmis laisse présager d'une grande diversité parmi les toxines (Hoffman, 2010). La probabilité de découvrir de nouvelles molécules d'intérêt pour l'homme n'en est que renforcée.

\section{Références}

Almunia, C., Bretaudeau, M., Held, G., Babon, A., 32 Marchetti, C., Castelli, F.A., Ménez, A., Maillere, B. 33 and Gillet, D. (2013). Bee Venom Phospholipase A2, a 34 Good "Chauffeur" for Delivering Tumor Antigen to the 35 MHC I and MHC II Peptide-Loading Compartments 36 of the Dendritic Cells: The Case of NY-ESO-1. PLoS 37 One, 8, e67645. 
Badr, G., Garraud, O., Daghestani, M., Al-Khalifa, M.S. and Richard, Y. (2012). Human breast carcinoma cells are induced to apoptosis by samsum ant venom through an IHF-1-dependant pathway, PI3K/AKT and ERK signaling; Cellular Immunology, 273, 10-16.

Bouzid, W., Klopp, C., Verdenaud, M., Ducancel, F. and Vétillard, A. (2013). Profiling the venom gland transcriptome of Tetramorium bicarinatum (Hymenoptera: Formicidae): The first transcriptome analysis of an ant species. Toxicon, 70:70-81.

Bouzid, W., Verdenaud, M., Klopp, C., Ducancel, F., Noirot, C. and Vétillard, A. (2014). De novo sequencing and transcriptome analysis for Tetramorium bicarinatum : a comprehensive venom gland transcriptome analysis from an ant species. BMC Genomics, 15, 987.

Davies, N.W., Wiese, M.D. and Brown, S.G.A. (2004) Characterisation of major peptides in "jack jumper" ant venom by mass spectrometry. Toxicon, 43, 173183.

Floren, A., Biun A. and Linsenmair, KE. (2002). Arboreal ants as key predators in tropical lowland rainforest trees. Oecologia 131, 137-144.

Fu, Y.J, Yin, L.T, Liang, A.H, Zhang, C.F, Wang, W., Chai, B.F, Yang, J.Y, and Fan, X.J. (2007). Therapeutic potential of chlorotoxin-like neurotoxin from the Chinese scorpion for human gliomas. Neurosci Lett, 412, 62-67.

Hoffman, D.R. (2010). Ant venoms. Curr Opin Allergy Clin Immunol, 10, 342-346.

King, GF. (2011). Venoms as a platform for human drugs: translating toxins into therapeutics. Expert Opin Biol Ther, 11, 1469-1484.

Lewis, RJ. and Garcia, ML. (2003). Therapeutic potential of venom peptides. Nat Rev Drug Discov, 2, 790-802

Liang, S. (2008). Proteome and peptidome profiling of spider venoms. Expert Rev Proteomics, 5(5):731-46.

Moon, D.O., Park, S.Y., Heo, M.S., Kim, K.C., Park, C., Ko, W.S., Choi, Y.H., and Kim, G.Y. (2006). Key regulators in bee venom-induced apoptosis are Bcl-2 and caspase-3 in human leukemic U937 cells through downregulation of ERK and Akt. Int Immunopharmacol, 6, 1796-1807.
Moreau, C.S., Bell, C.D., Vila, R., Archibald, B. and Pierce, N.E. (2006). Phylogeny of the ants: diversification in the age of Angiosperms. Science, 312, 101-104.

Pringos, E., Vignes, M., Martinez, J. and Rolland, V. (2011). Peptide Neurotoxins that Affect VoltageGated Calcium Channels: A Close-Up on $\omega$-Agatoxins. Toxins, 3, 17-42.

Ramezanpour, M., da Silva, K.B., and Sanderson, B.J. (2013).The effect of sea anemone ( $H$. magnifica) venom on two human breast cancer lines: death by apoptosis. Cytotechnology, 66, 845-852.

Ruiming, Z., Yibao M., Yawen, H., Zhiyong, D., Yingliang, W., Zhijian, C. and Wenxin, L. (2010). Comparative venom gland transcriptome analysis of the scorpion Lychas mucronatus reveals intraspecific toxic gene diversity and new venomous components. $B M C$ Genomics 11, 452.

Shin, J.M., Jeong, Y.J., Cho, H.J., Park, K.K., Chung, I.K., Lee, I.K., Kwak, J.Y., Chang, H.W., Kim, C.H., Moon, S.K., Kim, W.J., Choi, Y.H., and Chang, Y.C. (2013). Melittin suppresses HIF-1 $\alpha$ /VEGF expression through inhibition of ERK and mTOR/p70S6K pathway in human cervical carcinoma cells. PLoS One, 8, e69380.

Wang, W.X., Ji, Y.H. (2005). Scorpion venom induces glioma cell apoptosis in vivo and inhibits glioma tumor growth in vitro. J Neurooncol, 73, 1-7.

White, S.P., Scott D.L., Otwinowski Z., Gelb M.H. and Sigler P.B. (1990). Crystal structure of cobra-venom phospholipase A2 in a complex with a transition-state analogue. Science, 250, 1560-1563.

Zhu, L., Yuan, C., Chen, Z., Wang, W. and Huang, M. (2010). Expression, purification and characterization of recombinant Jerdonitin, a P-II class snake venom metalloproteinase comprising metalloproteinase and disintegrin domains. Toxicon, 55, 375-378. 